\title{
Polysèmes
}

Revue d'études intertextuelles et intermédiales

$10 \mid 2008$

Parcours / Détours

\section{Les couvertures de Step across this Line de Rushdie : entre fiction d'une non-fiction et balisage de parcours}

Simone Rinzler

\section{(2) OpenEdition \\ Journals}

Édition électronique

URL : http://journals.openedition.org/polysemes/629

DOI : $10.4000 /$ polysemes. 629

ISSN : 2496-4212

Éditeur

SAIT

Édition imprimée

Date de publication : 1 janvier 2008

Pagination : 73-90

ISSN : 0999-4203

\section{Référence électronique}

Simone Rinzler, «Les couvertures de Step across this Line de Rushdie : entre fiction d'une non-fiction et balisage de parcours », Polysèmes [En ligne], 10 | 2008, mis en ligne le 01 mars 2015, consulté le 30 avril 2019. URL : http://journals.openedition.org/polysemes/629; DOI : 10.4000/polysemes.629

Ce document a été généré automatiquement le 30 avril 2019.

Polysèmes 


\title{
Les couvertures de Step across this Line de Rushdie : entre fiction d'une non-fiction et balisage de parcours
}

\author{
Simone Rinzler
}

\begin{abstract}
"You shouldn't judge a book by its cover," snapped Blabbermouth [...]. In the first place, I wasn't "judging the book by the cover," as you suggested, because I could see all the pages-and in the second place, this isn't the "real world," not at
\end{abstract}

all. ${ }^{1}$

\section{Introduction}

1 Step across this Line, le recueil des textes de non-fiction post-fatwa de Salman Rushdie, interroge le rapport texte-image ( $\mathrm{T} / \mathrm{I}$ ou $\mathrm{T} \leftrightarrow \mathrm{I})$ tout autant que le rapport Auteur-Lecteur $(\mathrm{A} \leftrightarrow \mathrm{R})$.

2 Texte-fantôme, l'illustration de la première de couverture prend valeur de texte sans parole mais non sans langage, constituant pour le lecteur un parcours obligé qui oriente la lecture et l'interprétation du livre ainsi balisé. Texte anonyme à visée pragmatique répondant à des lois commerciales, l'illustration de couverture évoque une intertextualité brouillée et convoque la présence de l'éditeur en ghost-writer, auteur vampirique de l'auteur empirique pourtant seul signataire des textes imprimés.

3 Le dernier texte reproduit les conférences Tanner données à Yale où Salman Rushdie commente et révèle la portée politique d'une photographie de Sebastião Salgado ${ }^{2}$ (illustration 1).

4 Cette photo sert d'illustration de couverture pour la première édition (illustration 2). Toute trace en disparaît dans l'édition de poche britannique (illustration 3) comme dans 
la traduction française (illustration 4), limitant ainsi, non seulement la portée du texte lui-même, mais aussi la portée politique du recueil tout entier.

5 En effet, depuis cette édition originale, les illustrations de couverture prolifèrent, recontextualisant le recueil d'essais vers des lectures différentes, qui abrasent la force politique de l'engagement de Rushdie. Ces couvertures différentes, consensuelles, maquettées pour l'occasion, orientent le texte vers un marketing de masse, davantage que vers une prise de parole engagée, proposant une interprétation radicalement différente, et probablement moins subversive, en total contre-sens avec ma propre fiction de l'Auteur Rushdie et de son intention d'Auteur.

Dans la théorie ALTER de Lecercle (1999), le Texte (T) occupe la place centrale du dispositif, encadré par le Langage (L) et l'Encyclopédie (E), chacun conditionnant l'autre par un phénomène de réciprocité que l'on peut schématiser $\mathrm{L} \leftrightarrow \mathrm{T} \leftrightarrow \mathrm{E}$. Auteur (A) et Lecteurs (R) s'interpellent et se contre-interpellent par le biais du Texte (T) en fonction des représentations et des représentations d'intention que chacun se fait de l'autre acteur du dispositif pragmatique, ce que l'on peut représenter par le schéma contreinterpellatif: $A \leftrightarrow T \leftrightarrow R$. Le tout peut être très grossièrement schématisé ainsi: $\mathrm{A} \leftrightarrow \mathrm{L} \leftrightarrow \mathrm{T} \leftrightarrow \mathrm{E} \leftrightarrow \mathrm{R}^{3}$.

7 J'utiliserai la théorie ALTER de Jean-Jacques Lecercle pour interpréter pragmatiquement ces nouveaux rapports entre Auteur (A, comme Author) et Lecteur (R, comme Reader) par le biais du texte $(\mathrm{T})$ tenant compte du Langage (L) et de l'Encyclopédie (E) en y intégrant la notion selon laquelle certaines Images (I), quoique sans paroles, sont porteuses de Langage et d'Encyclopédie et fonctionnent comme de vrais Textes $(\mathrm{T})$ en tant qu'elles sont encodables et décryptables comme des Textes ( $T$ ), en suivant les phénomènes d'interpellation et de contre-interpellation entre leurs Auteurs (A) et leurs Lecteurs (R). Ceci donne l'équation $(\mathrm{T}=\mathrm{I})$ à l'intérieur de la nouvelle configuration $\mathrm{A} \leftrightarrow \mathrm{L} \leftrightarrow \mathrm{T}=\mathrm{I} \leftrightarrow \mathrm{E} \leftrightarrow \mathrm{R}$.

8 En dernière analyse, seule compte l'intention du Texte (T), indépendamment des intentions de l'Auteur (A) et du Lecteur (R) et des représentations qu'ils se font l'un de l'autre. Cependant, si le texte a une autonomie propre, celle-ci n'est pas immuable. En raison de modifications dues à des phénomènes de temporalité, un Texte $(\mathrm{T})$ de bonne facture, devient pérenne dès lors qu'il prouve sa capacité de recontextualisation à chaque nouvelle lecture dans le cadre d'une Encyclopédie (E) modifiée (En).

Quoique conçu pour "dire le canon littéraire », le modèle ALTER s'avère être un outil efficace d'interprétation de textes pragmatiques à visée politique. Peut-être doit-on y voir le fait que la théorie ALTER n'est pas qu'un outil d'interprétation pragmatique du fait littéraire, mais un véritable outil d'interprétation du texte militant de résistance et donc d'interprétation du fait politique. Grâce à la capacité de résistance que procure le travail du Langage et de l'Encyclopédie, c'est en tant qu'outil de résistance politique que la littérature est porteuse de réelle dimension politique, se prêtant au même type d'interprétation pragmatique que le texte engagé.

Dans un monde mouvant où le marketing déploie toute son énergie à récupérer la force d'évocation romantique du mot d'ordre révolutionnaire pour imposer de banals produits de consommation, l'étude de tout texte de résistance (qu'il soit littéraire, manifestaire, pamphlétaire ou philosophique) nécessite de s'intéresser à l'intention de l'Auteur, la (ou les) réception(s) du Texte par les Récepteurs/Lecteurs (R) ainsi qu'à la réception de l'intention de l'Auteur (A) telle qu'elle est fantasmée par ses Récepteurs/Lecteurs (R). 
11 Or, si l'on s'intéresse à l'intertextualité Texte/Image procurée par une couverture de livre, l'intention de l'Éditeur $\left(\mathrm{Int}_{\mathrm{Publsh}}\right)$ associée à l'intention du Marketeur/publicitaire $\left(\right.$ Int $\left._{\text {Mrkt }}\right)$ parasitent le modèle d'interprétation pragmatique ALTER à cinq places décrit par Jean-Jacques Lecercle. Par ailleurs, derrière l'intention de l'Éditeur se cache la Représentation que cet Éditeur se fait des intentions des actionnaires de la maison d'édition $\left(\right.$ Rep $\left._{\text {shrhld }}\right)$ ou plutôt $\left(\text { RepInt }_{\text {shrhld }}\right)^{4}$.

Face à des paramètres désormais si divers, il importe de s'en tenir au Texte lui-même, car il est, per se, porteur d'une intentionnalité propre qui échappe à l'intentionnalité des acteurs et $(\mathrm{R})$ des processus interpellatif et contre-interpellatif.

Or, la définition que Jean-Jacques Lecercle donne du texte littéraire canonique est en réalité une définition politique du texte engagé de bonne tenue stylistique dont le texte littéraire canonique est en fait un sous-genre, particulièrement apprécié, en raison de la capacité de résistance politique qu'offre la mise en discours proposée au Lecteur (R) par l'Auteur (A), travaillant conjointement Langage (L) et Encyclopédie (E) par le biais du Texte (T), dans un mouvement de va-et-vient ${ }^{5}$.

14 Si l'on applique la théorie ALTER à des textes de non-fiction comme les derniers essais de Rushdie (2002), écrits pendant les années de fatwa, on constate effectivement que la recontextualisation, l'engagement, le travail du Langage (L) et de l'Encyclopédie (E) (sous la forme $\mathrm{L} \leftrightarrow \mathrm{E}$ ) sont des actes de langage militants, porteurs de style au sens deleuzien du terme et font de ses textes de non-fiction de bonne tenue stylistique des textes canoniques au même titre que ses textes de fiction.

Lecercle subodorait que son modèle pouvait fonctionner parfaitement en dépit de l'absence de littérarité ou de fictionalité : «[1] [...] car je soutiens que la structure ALTER est la structure pragmatique de tout texte, et non seulement des textes littéraires $»^{6}$.

À la lumière des essais de Rushdie, il est possible d'affirmer que, non seulement le modèle fonctionne avec des textes non littéraires, mais qu'il est en réalité le modèle par excellence permettant de rendre compte de textes politiques revendicatifs dans la mesure où toute revendication d'identité implique que le mouvement althussérien d'interpellation et de contre-interpellation soit au cœur du dispositif énonciatif. Je fonde mon hypothèse à partir de la genèse du modèle ALTER. Le choix de Marx, Althusser et Deleuze comme mentors philosophiques dans l'élaboration d'une théorie du littéraire inspirée d'une théorie du langage devait nécessairement amener son concepteur à envisager une théorie d'où le politique ne pourrait en aucun cas être évacué. Mais il va de soi, par ailleurs, que l'on ne peut négliger non plus l'influence de Jakobson, d'Eco, de Bakhtine et d'Austin comme inspirateurs linguistiques, littéraires et pragmatiques.

En dépit des interrogations du petit Haroun : "What's the use of stories that aren't even true? $»^{7}$, la défense du droit à l'existence de la littérature est un devoir politique. Tout comme il est un devoir de défendre le respect du Texte (T) qui ne doit subir aucune altération autre que celle souhaitée par l'Auteur (A), que ce texte soit littéraire ou non.

Cette défense est primordiale, à une époque où l'édition semble être parfois davantage mue par des impératifs économiques que par le contenu des textes. Entre marché de l'édition et plaisir du texte, le choix est facile à effectuer. Pour ma part, je me place résolument du côté de la défense du Texte, quel qu'il soit, et du côté de la défense d'un accès égalitaire à l'éducation, la culture, les sciences et les arts ${ }^{8}$. 


\section{Intentions et recontextualisations : le photographe et l'auteur}

\section{Intention politique du photographe}

Cette photographie, emblématique de la notion de passage de frontière, sans l'explication d'accompagnement fournie dans l'album, est trompeuse. Qu'y voit-on ? Parodiant Daniel Arasse, on pourrait dire qu'on n'y voit rien, ou du moins, pas grand-chose. La photo est un peu floue. Loin d'être le cliché le plus esthétique de Salgado, ce n'en est pas pour autant le moins significatif. Dans un paysage désertique bordé à gauche de maisons individuelles et 
de cabanes de fortune, une voiture roule à vive allure à en juger par le nuage de poussière qui se dégage dans son sillage dans la partie non construite, sur la moitié droite de la photo. Le blanc de la poussière du désert remplit une grande partie de l'espace de la page 1 de couverture (illustration 2). Au point où il n'est pas évident de remarquer la petite silhouette noire d'un homme au pas de course tout à fait en bas à gauche du livre. En lecteurs habitués à lire de gauche à droite et de haut en bas, notre champ de vision s'organise selon un axe allant d'un point en haut à gauche à un point en bas à droite, ce qui nous fait souvent ignorer au premier coup d'œil les éléments isolés situés en bas à gauche d'une image ${ }^{14}$. La photo, paradoxale, ne se laisse pas décrypter sans le mode d'emploi de son auteur. Mais pour le lecteur de Rushdie ignorant le travail de Salgado, le décryptage de la couverture ne peut intervenir que lors de la lecture du tout dernier texte. Dès lors, la photo un peu terne prend une valeur tout autre. Rushdie reprend le commentaire de Salgado à destination du lecteur avec, il faut bien le noter, des imprécisions identiques à celles du narrateur de Midnight's Children.

\section{Intention politique de Salman Rushdie conférencier et essayiste}

Dans Step across this Line, Rushdie fait œuvre de critique littéraire, d'analyste politique, de militant des Droits de l'Homme, mais aussi de philosophe dans la manière dont il retravaille le concept de la Frontière pour revendiquer la nécessité de transgresser toutes les frontières (physiques, littéraires, politiques, religieuses et philosophiques). Le voyage comme quête façonne celui qui s'y soumet, que cela soit volontairement ou par force (exil politique ou économique) :

The intellectual uprooted against his will (a category that includes, these days, many of the finest Arab writers) rejects the narrow enclosures that have rejected them. There is great loss, and much yearning, in such rootlessness. But there is also gain. The frontierless nation is not a fantasy. ${ }^{15}$

Dans sa proposition d'une nouvelle théorie de la post-frontière, Rushdie s'appuie sur différentes sources encyclopédiques qui entrent en résonance intertextuelle les unes avec les autres : la Conférence des oiseaux du poète soufi Fariduddin Attar, le roman de sciencefiction de Doris Lessing The Making of the Representative for Planet 8, la légende du Roi Arthur et la quête du Graal, le poème de Cavafy En attendant les Barbares, bien sûr Alice, sans oublier la photo de Salgado dont il sélectionne et commente le cliché ainsi :

There is a photograph by Sebastião Salgado that shows the wall between the United States and Mexico snaking over the crests of hills, running away into the distance, as far as the eye can see, part Great Wall of China, part Gulag. There is a kind of brutal beauty here, the beauty of starkness. At intervals along the wall there are watchtowers, and these so-called sky-towers are manned by armed men. In the photograph, we can see the tiny silhouetted figure of a running man, an illegal immigrant, being chased by other men in cars. The strange thing about the picture is that, although the running man is clearly on the American side, he is running toward the wall, not away from it. He has been spotted, and is more afraid of the men bearing down on him in cars than of the impoverished life he thought he had left behind. He's trying to get back, to unmake his bid for freedom. ${ }^{16}$ en 2002, ce qui en infléchit le propos:

So freedom is now to be defended against those too poor to deserve its benefits by the edifices and procedures of totalitarianism. What kind of freedom is it, then, that we enjoy in the countries of the West-these exclusive, increasingly well-guarded 
enclaves of ours? That is the question the photograph asks, and before September 11, 2001, many of us-many more I suspect, than today-would have been on the running man's side. ${ }^{17}$

\section{d'engagement. Il n'en est en fait rien :}

Think for a moment about this image of a running man, a man who has nothing, who is no danger to anyone, fleeing the land of the free. For Salgado, as for myself, the migrant, the man without frontiers, is the archetypal figure of our age. Salgado has spent many years among the world's displaced peoples, the uprooted and the re-rooted, chronicling their border crossings, their refugee camps, their desperations, their ingenuities: creating an extraordinary photographic record of this most important of contemporary phenomena. The pictures show that there has never been a period in the history of the world when its peoples were so jumbled up. We are so thoroughly shuffled together, clubs among diamonds, hearts among spades, jokers everywhere, that we're going to have to live with it. In the United States, this is an old story. Elsewhere, it's a new one, and it doesn't always go down well. As a migrant myself, I have always tried to stress the creative aspects of such cultural commingling. The migrant, severed from his roots, often transplanted into a new language, always obliged to learn the ways of a new community, is forced to confront the great questions of change and adaptation; but many migrants, faced with the sheer existential difficulty of making such changes, and also, often, with the sheer alienness and defensive hostility of the peoples among whom they find themselves, retreat from such questions behind the walls of the old culture they have both brought along and left behind. The running man, rejected by those people who have built great walls to keep him out, leaps into a confining stockade of his own. ${ }^{18}$

Dans son commentaire de la photo de Salgado, Rushdie fait fonctionner le modèle ALTER ainsi : Rushdie Auteur (A) de la conférence et en même temps Lecteur (R) de la photo de Salgado (Rush $\mathrm{A}_{\text {Lect }} \& \mathrm{R}_{\mathrm{Phot}}{ }^{19}$ ) se fait une représentation de Salgado-Auteur-de-la-Photo $\left(\operatorname{Rep}_{\mathrm{APhot}}\right)$. À partir de sa propre interpellation créée par ce Texte-en-Image (Tinl) ${ }^{20}$, Rushdie Auteur (A) crée pour les Lecteurs (R) de son Texte $(\mathrm{T})$ une nouvelle interpellation par le biais de son texte, laissant le Lecteur (R) libre d'être interpellé (à la fois) par ce nouveau Texte-en-Mots (TinW) en se faisant une double représentation des deux Auteurs (A) : Salgado Auteur $(A)$ du Texte-en-Image $\left(\mathrm{A}_{\text {Tinl }}\right)$ et Rushdie Auteur (A) du Texte-en-Mots $\left(\mathrm{A}_{\mathrm{TinW}}\right)$.

\section{Recontextualisation de la photographie de Salgado dans le cadre du dernier essai du recueil}

Décrite et explicitée dans la conférence, la photographie contribue à construire (et à limiter) le concept de l'injonction à transgresser toutes les frontières contenue dans le titre du livre. Les deux éditions reproduisant le texte sans son illustration recontextualisent le Texte-en-Mots en l'amputant d'une grande partie de son potentiel d'intertextualité Texte/Image (T/I) alors même que les citations littéraires, politiques ou philosophiques sont maintenues, laissant le reste du Texte-en-Mots (TinW) intact.

Le lecteur de Rushdie intrigué qui découvre les projets de Salgado (des Textes-en-Images accompagnés de Textes-en-Mots) peut alors effectuer une nouvelle lecture recontextualisée du Texte-en-Mots de Rushdie, Texte $(\mathrm{T})$ travaillé par l'intertextualité Texte/Image (T/I) grâce à la recontextualisation offerte par cet apport d'une Encyclopédie (E) élargie. 


\section{Revendication de l'intention politique de l'édition originale}

L'éditeur de la première édition, Random House, en choisissant (ou en acceptant) la photo de Salgado en couverture, revendique lui aussi l'intention politique de Rushdie (et de Salgado, par la même occasion) puisqu'il la fait sienne en la publiant, donc sans dénaturer la pensée de Rushdie qui s'appuie longuement sur cette image comme intertexte. La photo est partie intégrante du texte et du message rushdiens. De la même manière que le texte du poème de Cavafy est reproduit dans le corps de la conférence finale, l'image, peut-être inconnue des lecteurs, n'est pas seulement décrite (en une ekphrasis imparfaite), mais est intégralement reproduite, si ce n'est que le paratexte de la couverture en cache certains éléments (illustration 2). Par ailleurs, on ne voit que la partie droite du cliché quand le livre est fermé. Certes il n'y a aucun montage : il s'agit bien de la photographie intégrale, mais dont seule la partie droite se donne à voir quand le livre est fermé, ce qui en change le point focal ou la ligne du fuite.

\section{Contextes et recontextualisations : l'auteur et l'éditeur}

\section{Élaboration du concept de la post-frontière à Yale}

Les deux conférences de Yale éclairent la portée politique de tout le recueil. Elles bénéficient d'un contexte particulier puisqu'elles permettent à Rushdie de continuer à revendiquer publiquement son engagement. Elles ont eu lieu à un moment politique qui modifie la position de Rushdie par rapport à son nouveau concept, la théorie de la postfrontière :

Time, perhaps, to propose a new thesis of the post-frontier: to assert that the emergence, in the age of mass migration, mass displacement, globalized finances and industries, of this new, permeable post-frontier is the distinguishing feature of our times, and, to use Turner's phrase ${ }^{21}$, explains our development as nothing else can. For all their permeability, the borders snaking across the world have never been of greater importance. This is the dance of history in our age: slow, slow, quick, quick, slow, back and forth and from side to side, we step across these fixed and shifting lines. ${ }^{22}$

En effet, ces conférences américaines ont lieu après le 11 septembre 2001 dont on voit bien l'influence sur les engagements de Rushdie.

La teneur du texte éponyme, dont l'injonction paradoxale Step across this Line infléchit la lecture, de même que la photographie de Salgado qui en est un des objets, permettent à Rushdie de développer son concept de la transgression de frontières. Or, selon Deleuze, le travail du philosophe est la création de concepts. En nous proposant sa vision conceptualisée du monde, Rushdie amorce ici son « pas philosophique ${ }^{23}$.

Pour moi, Step across this Line va bien au-delà de la simple collection d'articles des années noires. Il s'agit d'une œuvre complète qui permet à Rushdie d'effectuer sa propre revendication d'identité. Celle-ci peut être considérée comme performative car effectuée à la première personne et au présent. En tant que manifeste de revendication identitaire de son auteur, le Texte (T) prend une valeur performative par le seul fait de son existence dans l'espace public. 


\section{Contexte original de la première publication du recueil Step across this Line}

Avec cette publication, Rushdie déclare vouloir en finir définitivement avec la fatwa. Ainsi, non seulement il fait le point sur ses positions philosophiques et littéraires, mais il escompte bien que ce recueil pourra être son dernier mot non fictionnel en relation avec l'Affaire. Ainsi qu'il le déclare, Rushdie choisit de retourner à la littérature :

But as well as fighting the fight, which I will surely go on doing, I have grown determined to prove that the art of literature is more resilient than what menaces it. The best defense of literary freedoms lies in their exercise, in continuing to make untrammelled, uncowed books. So, beyond grief, bewilderment, and despair, I have rededicated myself to our high calling. ${ }^{24}$

Comme souvent chez les exilés, le langage, et plus particulièrement le langage de la fiction, devient la seule patrie et c'est en cela que le langage du nomade, de l'exilé, du déraciné, du Juif errant ou de l'Indien plusieurs fois émigré et des migrants du XXe siècle (qu'ils soient photographiés ou non par Salgado) sont le fondement de sa production de romancier et d'essayiste.

\section{Couverture du « paperback » et édulcoration du message politique}

Dans l'édition de poche anglaise, la photographie a non seulement disparu de la couverture, mais elle a aussi totalement disparu de l'ouvrage. Le Texte-Livre tout entier en subit une recontextualisation qui en amoindrit la portée politique. Certes, on pourra gloser en signalant que l'ekphrasis rushdienne devrait suffire. Il n'en demeure pas moins vrai que pour le lecteur de l'édition originale, cette photo énigmatique avait fini par faire sens. L'interrogation du lecteur cessait quant au choix d'une couverture si peu engageante pour se transfigurer en véritable épiphanie de lecture: à défaut d'être engageante, la photo était engagée.

Avec la photo mise en scène du paperback, on a affaire à une couverture maquettée pour un marché d'acheteurs de biens culturels (illustration 3). Dans le dispositif pragmatique à visée économique mis en place par l'éditeur, ce n'est pas à une Représentation de Lecteurs rushdiens ( Rep $_{\text {RRush }}$ ) mais à une Représentation de Consommateurs ou d'Acheteurs $\left(\operatorname{Rep}_{\text {Consu }}{ }^{25}\right)$ que l'on a affaire.

Qu'y voit-on? La photo en noir et blanc d'un crayon à papier muni d'une gomme à l'une de ses extrémités et dont la mine de graphite a été remplacée par une extrémité d'allumette déjà consumée. Le crayon tient en équilibre, posé verticalement sur sa gomme sur un plan gris indéterminé (table ou sol, on ne sait guère). Le fond ne nous est pas d'un grand secours: derrière ce plan horizontal, le fond vertical est blanc, neutre, vide. Le crayon, court, a déjà beaucoup servi : il en reste moins de la moitié. La métaphore visuelle de l'allumette brûlée introduit une connotation négative, comme si la mine, entièrement consumée symbolisait la flamme de l'écriture éteinte. La mine inutilisable devient dérisoire.

41 Seule touche de couleur, en jaune paille foncé, le prénom et le nom de l'auteur en lettres capitales suivi en dessous du titre en capitales en deux couleurs, rouge d'une part, et 
framboise clair pour le mot transgressif across qui, imprimé dans un ton plus clair, est nettement moins visible que le titre, déjà assez peu lisible sur le fond gris. Tout se passe comme s'il était question de gommer la force transgressive de l'injonction du titre. En revanche, "Collected non-fiction 1992-2002", quoique en capitales de plus petites dimensions, est plus lisible car la différence de ton est plus importante entre le fond et les lettres que pour le titre principal. On peut se demander s'il n'y a pas eu une forme de gommage volontaire du titre subversif en plus du gommage de l'image à message politique avec cette mise en scène du paratexte éditorial.

De plus, la recommandation du Financial Times cautionne la publication, argument paratextuel éditorial pour décider pour l'acheteur hésitant :

Over the quick sprint of an essay,

Rushdie dazzles and swoops.

Financial Times

Enfin, l'éclairage du vrai-faux crayon vient de la droite, laissant les ombres à gauche de la photographie. Dans notre représentation habituelle sur une surface plane, la gauche représente le passé et la droite l'avenir. Ici, le sombre passé est dans l'ombre, à gauche. En revanche, la partie droite du crayon est saturée de lumière. Avec cet éclairage par la droite, on entre dans le livre par l'illustration. Une certaine connotation positive est donc récupérée par l'orientation de l'éclairage du sujet photographié pour la circonstance.

\section{Compromis de la couverture de l'édition française}

\section{Illustration de couverture}

Avec le sens interdit de la couverture française, on remplace la photo authentique par un dessin de type cartoon (illustration 4). Le nom de l'auteur est en minuscules, sauf pour les initiales. Les caractères sont de très sobres lettres noires, d'assez petite taille. Le titre en rouge vif est assorti au rouge du grand panneau de « sens interdit » symbolisé par l'ellipse de disque rouge dont la barre horizontale blanche a été remplacée par un creux, un peu comme dans un dessin d'Escher. La barre blanche qui traverse le sens interdit ne s'inscrit pas sur une surface plane. Elle représente un évidement dans la surface du disque rouge, lui conférant une épaisseur en trois dimensions. À la gauche de cette barre blanche, donnant du relief à cette surface plane, les marches grises et ombrées d'un escalier descendant vers les profondeurs invitent au rappel de la situation de Rushdie contraint à la clandestinité, suggérant une intertextualité entre la version française et l'expression anglaise to go underground.

\section{Titre et traduction de la métaphore transgressive : quelques aspects linguistiques}

De l'injonction négative bien connue en anglais « Do not step across this line», ordre absolu émanant d'une autorité souvent mal définie, mais indiscutable, Rushdie se joue en passant à l'injonction à polarité positive avec son titre qui invite son Lecteur $(\mathrm{R})$ à une transgression joyeuse sous la forme impérative: "Step across this line». L'absence de ponctuation en anglais n'est pas neutre. L'injonctive Step across this line non ponctuée d'un point d'exclamation permet de donner au lecteur un parcours de lecture qui est en fait une proposition d'interprétation pragmatique dans laquelle le lecteur reste libre de son 
choix d'obéir ou non à l'injonction suggérée, là où un point d'exclamation aurait pu prendre une connotation autoritaire imposant un ordre.

La traduction du titre en français Franchissez la ligne..., associée à la perte de sens liée au choix d'illustration de couverture, contribue à affaiblir la teneur du message politique. Je n'en ferais pas reproche au traducteur, tant il est vrai que le passage de la frontière d'une langue à une autre nécessite bien souvent des glissements sémantiques ${ }^{26}$.

L'aposiopèse, présente sous la forme de points de suspension, met davantage en valeur la possibilité d'interprétation du Lecteur (voire une dernière ironie de Rushdie) que l'intention de l'Auteur (IntA) sous la forme de programme politique, littéraire et philosophique que celui-ci propose, sans l'imposer, à son Lecteur (R).

Dans le titre en anglais, malgré l'impératif, il n'y a aucune ponctuation, ce qui est une pratique courante en matière de titres. Cette absence de ponctuation n'est pas neutre dans la mesure où le titre est une injonctive à l'impératif qui reprend, pour le détourner, un cliché négatif bien connu grâce à un changement de polarité. Or une injonctive peut s'accompagner d'un point d'exclamation, même sur une couverture. Ni Rushdie ni son éditeur n'ont choisi cette proposition plus autoritaire. Quand une injonctive apparait dans un titre de livre accompagnée d'un point d'exclamation, la part interprétative du Lecteur (R) en est amoindrie puisque le dispositif contre-interpellatif est fermé. Le Lecteur $(\mathrm{R})$ ne semble pas autorisé à désobéir à l'injonction et ne peut que s'exécuter en y répondant positivement. Dans le titre français, les points de suspension constituent déjà un commentaire du titre. La liberté interprétative du lecteur francophone est placée sous le contrôle de ce commentaire ambigu: s'agit-il d'une simple suggestion ou d'une dernière ironie rushdienne ? Il incombe au lecteur francophone $\left(\mathrm{R}_{\mathrm{Frch}}\right)$ d'en décider, mais cette ambiguïté n'existait pas dans les éditions anglaises pour le lecteur anglophone $\left(\mathrm{R}_{\mathrm{Engl}}\right.$ ) .

Enfin, l'éditeur de la traduction française se fait une représentation d'un lectorat francophone peu concerné par la question du passage de la frontière mexicanoaméricaine. Ainsi qu'il le stipule: "Avec l'accord de l'auteur, quelques textes, d'un moindre intérêt pour le public européen, ont été écartés de l'édition française $»^{27}$.

J'ajouterai notamment le texte d'ouverture Out of Kansas sur le film The Wizard of $\mathrm{Oz}$, fondamental dans la thématique rushdienne ${ }^{28}$. Mais il n'est aucunement mentionné que la photo de couverture est différente de celle de l'édition originale. Peut-être y a-t-il eu un problème de droits de reproduction à l'époque? Il ne m'appartient pas de mener l'enquête à ce sujet car ce qui m'intéresse ici, c'est d'analyser en quoi, en proposant un Texte-en-image différent, l'Éditeur et donc, implicitement au moins, l'Auteur proposent un texte différent ${ }^{29}$.

\section{Conclusion}

51 Entre texte auctorial et texte éditorial, entre œuvre créatrice et marketing éditorial d'une industrie commerciale, la couverture porte une intertextualité et une hybridité pragmatiques qui la rendent parfois difficile à interpréter, voire à accepter. L'insertion d'acteurs commerciaux plus ou moins anonymes dans le processus de création littéraire ou scriptural brouille le message envoyé au lecteur (R) par l'auteur (A), balise son parcours de lecture le plus souvent en proposant, non pas une intertextualité riche de résonances, mais une interprétation simpliste qui déclasse l'ouvrage qui est ainsi, non pas 
«habillé », mais déshabillé, dénudé, privé de son potentiel interprétatif, une nouvelle fiction dont l'Éditeur devient l'Auteur $\left(\right.$ Publsh $\left._{\mathrm{A}}\right)$.

Tout lecteur sait que son parcours de lecture s'accompagne de signes-en-image qui accompagnent le texte. Il sait aussi que certains écrits et certaines lectures restent attachées au parcours de lecture : au souvenir du lieu et du moment de lecture, qui eux aussi recontextualisent chaque lecture de chaque lecteur, s'ajoute un parcours sensuel (toucher, odeur du livre), mais aussi un parcours esthétique (couleur, forme, format). Fait de plus en plus développé depuis que l'on a affaire à une véritable industrie du livre, couleurs, images, photos, reproductions artistiques créditent l'œuvre d'une dimension esthétique associée qui n'a pas été nécessairement choisie, voire souhaitée, par l'auteur dont l'intentionnalité auctoriale se trouve irrémédiablement brouillée par ces ajouts éditoriaux paratextuels. Le choix de l'illustration de couverture, de la mise en page, du paratexte de couverture, les couvertures des livres que nous lisons ne sont pas neutres. Elles balisent nos parcours de lectures, ajoutant un paramètre éditorial non prévu par ALTER qui, le plus souvent, parasite le travail de l'Auteur (A) en s'interposant délibérément entre l'Auteur $(\mathrm{A})$, le Texte $(\mathrm{T})$ et le Lecteur $(\mathrm{R})$, proposant ainsi une interprétation fermée préformatée du Texte $(\mathrm{T})$ ainsi emballé, maquetté, marketé. Ces éléments paratextuels ne sont pas dépourvus de pouvoir pragmatique. Capables du meilleur comme du pire, ils accompagnent notre lecture tout autant que le choix du titre dont on sait bien qu'il échoit souvent à d'autres que l'auteur empirique lui-même. Situés à mi-chemin entre propagande publicitaire et intertextualité foisonnante, tous n'ont pas la même valeur ${ }^{30}$. Il est de notre devoir de le signaler dans l'espoir que les éditeurs finissent par comprendre que les lecteurs ne souhaitent pas que les textes originaux soient altérés, voire déclassés, par des préoccupations mercantiles. Loin de moi l'idée de m'insurger contre toutes les couvertures, bien au contraire. Elles font partie de mon expérience de lectrice et participent au travail d'interpellation que l'objet-livre effectue à mon égard, et ce travail n'est pas pour me déplaire dans de nombreux cas.

Mais quand la politique éditoriale amoindrit ce que je considère comme un texte majeur de résistance politique, il n'est pas nécessaire de la cacher, je suis un chercheur en humanités bien en colère et je vois rouge! Mais peut-être n'est-ce là qu'un effet pervers de la couverture française voire, qui sait? de certaines opinions...

\section{BIBLIOGRAPHIE}

Arasse, Daniel. On n'y voit rien - Descriptions. Paris : Denoël, coll. Folio/Essais, 2000.

Hutcheon, Linda. The Politics of Postmodernism (1989). London: Routledge, coll. New Accents, 2002.

Lecercle, Jean-Jacques. Interpretation as Pragmatics. New York: Saint Martin’s Press, 1999.

Lecercle, Jean-Jacques \& Ronald Shusterman. L'Emprise des Signes - Débat sur l'expérience littéraire. Paris : Seuil, coll. Poétique, 2002.

Milner, J-C. Le Pas philosophique de Roland Barthes. Lagrasse : Verdier, coll. Philia, 2003. 
Rushdie, Salman. Haroun and the Sea of Stories. London: Granta books published in association with the Penguin Group, 1990.

Rushdie, Salman. Imaginary Homelands, London: Granta Books in association with Penguin Books, 1992.

Rushdie, Salman. Step across this Line - collected nonfiction 1992-2002. New York: Random House, 2002.

Rushdie, Salman. Step across this Line - collected nonfiction 1992-2002. London: Vintage, Random House, 2002-3.

Rushdie, Salman. Franchissez la ligne... - Essais 1992-2002. Traduit de l'anglais par Philippe Delamare. Paris : Plon, 2003.

Rushdie, Salman. Step across this Line - collected nonfiction 1992-2002. New York: Modern Library Paperbacks, Random House, 2002-3.

Salgado, Sebastião. Une Certaine Grâce. Textes de Eduardo Galeaono et Fred Ritchin. Paris : Nathan/Image, 1990.

Salgado, Sebastião. Terra. Introduction de José Saramago. Poèmes de Chico Buarque. Paris : Éditions de La Martinière (non cité), 1997.

Salgado, Sebastião. Exodes - Humanité en mouvement - 1993-1999. Paris : Éditions de La Martinière, 2000.

Salgado, Sebastião. Migrations - Humanity in transition 1993-1999. New York: Aperture Foundation, 2000.

Sites Web consacrés à Salgado:

Site Terra.com : http://www.terra.com.br/sebastiaosalgado/

(dernière consultation du site le 27 juin 2004)

Site University of California, Berkeley, section consacrée à Salgado : http://www.berkeley.edu/ news/media/releases/2002/01/18_salgado.html

Liste des sites renvoyant aux illustrations de Step accross this Line de Salman Rushdie

http://www.amazon.com/Step-Across-This-Line-Nonfiction/dp/0679463348 (Photo de l'édition originale du livre de Rushdie)

http://www99.epinions.com/content_117142359684 (photo de la nouvelle édition de poche reprenant la photo originale de Salgado)

http://www.fantasticfiction.co.uk/r/salman-rushdie/step-across-this-line.htm (édition de poche avec le crayon / allumette brûlée (photo assez floue)

http://www.redroom.com/publishedwork/step-across-line-collected-nonfiction-1992-2002 (photo de la nouvelle édition de poche reprenant la photo originale de Salgado)

http://www.amazon.fr/Franchissez-ligne-Salman-Rushdie/dp/2259193447 (couverture de la première édition de la traduction française) 


\section{NOTES}

1. Rushdie, 1990, 114.

2. San Ysidro, California, July 1997.

3. Il va de soi qu'une représentation aussi succincte ne peut être que réductrice.

4. Les abréviations en indice correspondent à la traduction anglaise des termes publisher, marketer et shareholder.

5. Selon Lecercle, le texte canonique est pérenne car recontextualisable et autorise que le modèle ALTER fonctionne à plein.

6. Lecercle \& Shusterman, 2002, 109.

7. Rushdie, 1990, 22 et 27.

8. Ce sont les valeurs de la République française rappelées par la fresque sculptée au fronton de la Mairie de Puteaux dans les Hauts de Seine.

9. The Creation of a global proletariat (cf sites internet consacrés à Salgado).

10. Site internet terra.com

11. Ibid.

12. Salgado, 2000 : fascicule explicatif, 2.

13. Le lien entre Salgado et Rushdie n'échappera pas au lecteur de The Ground Beneath her Feet dont le narrateur est un photographe qui, par certains côtés peut faire penser à Salgado tel qu'on se le représente au travers de ses recueils de photographies et de son site internet.

14. C'est pour cette raison que les publicités au bas des pages de gauche d'une revue sont moins chères pour l'annonceur que celles situées en bas des pages de droite. Nous voici, à nouveau, rattrapés par le marketing.

15. Rushdie, 2002, Notes on Writing and the Nation, section 8,61 .

16. Rushdie, 2002, 354-355.

17. Rushdie, 2002, 355.

18. Ibid, 356

19. La conférence (« Lecture » en anglais) a été abrégée en Lect dans la notation utilisée ici.

20. Qui est aussi une «image-en-texte » (IinT) comme me l'a très justement fait remarquer Liliane Louvel que je remercie ici.

21. "According to the Turner thesis: the existence of an area of free land, its continuous recession, and the advance of American settlement westward, explain American development... [which] has exhibited not merely advance along a single line, but a return to primitive conditions on a continually advancing frontier line, and a new development for that area. American social development has been continually beginning over again on the frontier. This perennial rebirth, this fluidity of American life, this expansion westward with its new opportunities, its continuous touch with the simplicity of primitive society, furnish the forces dominating American character. Turner characterizes the frontier as "the meeting point between savagery and civilization," a formulation that will not endear him to a more culturally sensitive modern audience » (Rushdie, op. cit., 2002, 360).

22. Rushdie, 2002, 365.

23. Milner, 2003.

24. Rushdie, 2002, February 1999: Ten Years of the Fatwa, 266. Originellement, l'article s'intitulait Unfunny Valentine.

25. L'abréviation Consu en indice est mise ici pour consumer.

26. Au-delà de cette limite, votre ticket devient valable est une suggestion possible.

27. Rushdie, 2003, édition française, page éditoriale non numérotée. 
28. Rushdie y explique que sa première expérience de littérarité passe par la découverte de ce film, que, devenu adulte, il continue d'admirer avec des yeux d'enfants, mais aussi avec un regard aiguisé de critique et de philosophe.

29. Depuis la rédaction de cette communication, j'ai eu connaissance d'une nouvelle édition de poche publiée à New York en 2003 par Random House reprenant la photo originale de Salgado agrémentée d'un travail de maquettage de la couverture: une fine ligne jaune barre verticalement le verso de la reliure ainsi que la photographie de Salgado en première page de couverture. La découverte tardive de cette édition de poche américaine ne me permet pas d'en effectuer l'étude détaillée. On notera cependant que l'on a affaire à une couverture de compromis entre maquettage éditorial et fidélité intertextuelle, ce qui est, à mon sens, un moindre mal. La matérialité de la ligne jaune constitue un nouveau balisage de parcours de lecture forcé pour un lectorat décidément considéré bien inepte si l'on en juge par cette politique éditoriale imposant un tel commentaire paratextuel.

30. Ils sont aussi un argument de vente dans lequel la couverture reste le dernier appel de l'éditeur auprès du lecteur et constitue la frontière entre l'achat ou le non-achat du livre. Ceci concerne aussi les emprunts ou le non-emprunt en bibliothèque, car désormais, les bibliothèques ne masquent plus le résultat du travail éditorial des couvertures par une couverture anonyme comme ce fut longtemps le cas.

INDEX

oeuvrecitee Step across this Line 